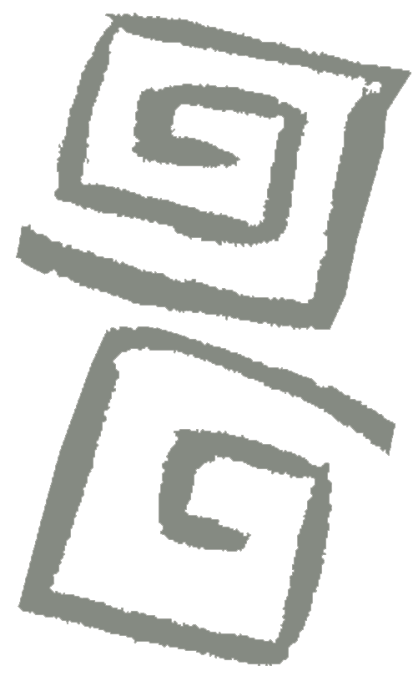

\title{
Medicalización psiquiátrica en tres prisiones femeninas brasileras: un abordaje etnográfico sobre los itinerarios de criminalización, patologización y farmacologización
}

\author{
Psychiatric medicalization in three Brazilian women's \\ prisons: an ethnographic approach to the itineraries of \\ criminalization, pathologization and pharmaceuticalization
}

Laura Ordoñez-Vargas ${ }^{1}$, Claudia Margarita Cortés-García

${ }^{1}$ Autor de correspondencia. Doctora en Antropología Social. Docente e investigadora, Escuela de Ciencias Humanas, Universidad del Rosario, Bogotá, Colombia. $\square$ (iD)

${ }^{2}$ Doctora en Salud Pública. Docente e investigadora, Escuela de Ciencias Humanas, Universidad del Rosario, Bogotá, Colombia. $\triangle$ iD
RESUMEN Este artículo analiza los procesos de medicalización psiquiátrica de las mujeres dentro de tres prisiones femeninas brasileñas, desde los campos de la salud mental, la antropología del confinamiento y los estudios de género. Se llevó a cabo un estudio cualitativo de carácter etnográfico (de octubre de 2006 a febrero de 2007) con observación participante, conversaciones informales, entrevistas semiestructuradas y relatos de vida. Entendidos como dispositivos estatales de poder, los procesos de medicalización psiquiátrica están marcados por tres itinerarios: criminalización masiva de mujeres, patologización (de la criminalidad femenina) y farmacologización psiquiátrica (en especial, a través de la administración de fármacos psicoactivos). Estos procesos instauran un círculo vicioso que perpetúa o produce los trastornos psiquiátricos que pretende curar, alimentando el uso y consumo de drogas (ilícitas-lícitas-ilícitas) y su vinculación con la ilegalidad, y permiten ver cómo el género, intersectado con otras categorías de diferenciación, influye en la producción de enfermedades mentales, las cuales deben ser tratadas como un problema de salud pública que se extiende más allá de los muros de las prisiones femeninas.

PALABRAS CLAVES Prisiones; Mujeres; Medicalización; Uso Excesivo de Medicamentos Recetados; Salud Mental; Psicotrópicos; Brasil.

\begin{abstract}
This article analyzes the psychiatric medicalization of women in three Brazilian women's prisons, from the fields of mental health, the anthropology of confinement, and gender studies. A qualitative study employing ethnographic methods was carried out from October 2006 to February 2007, which included participant observation, informal conversations, semi-structured interviews, and life histories. Understood as a state device of power, psychiatric medicalization processes are marked by three itineraries: the mass criminalization of women, pathologization of women's crimes, and psychiatric pharmaceuticalization (especially through the administration of psychoactive drugs). These processes thus establish a vicious cycle that perpetuates or produces the disorders that psychiatry seeks to heal and enables the continuity of drug use (illicit-licit-illicit) and its ties to the illegal drug market, while highlighting how gender - in its intersections with other categories of differentiation - influences the production of mental illnesses, which must be treated as a public health problem that extends beyond the walls of women's prisons.
\end{abstract}

KEY WORDS Prisons; Women; Medicalization; Prescription Drug Overuse; Mental Health; Psychotropic Drugs; Brazil. 


\section{INTRODUCCIÓN}

Durante el desarrollo de una investigación dentro de la Penitenciaria Femenina del Distrito Federal (PFDF), en Brasilia (desde abril a octubre de 2004), sobre el papel de la religión intramuros, nos sorprendió encontrar que la vida diaria de las prisioneras en las cárceles estaba marcada por la frecuente y alta ingesta de drogas psiquiátricas o "remedios controlados", como los Ilamaban las mujeres privadas de la libertad. Fueron las marcas físicas, visibles y vehementemente inscritas en los cuerpos de las mujeres encarceladas (ojos rojos, miradas perdidas, cuerpos hinchados, piel brotada, entre otras), las que sin buscarlo nos informaron sobre esta práctica cotidiana y naturalizada en las prisiones femeninas. Había mujeres que, durante el trabajo de campo, consumían dos o tres veces al día diversas combinaciones de estos medicamentos (ansiolíticos, antidepresivos, antipsicóticos, anticonvulsivos, entre otros), independientemente del tiempo que llevaran presas. Este hallazgo nos interpeló a realizar una investigación posterior (desde octubre de 2006 a octubre de 2008), enfocada particularmente en los itinerarios de farmacologización psiquiátrica que las mujeres inician desde el momento en que son privadas de la libertad, de la cual se deriva la reflexión que realizaremos en este artículo.

Para comenzar este artículo, es necesario entender el carácter generizado del castigo estatal $^{(1)}$, con el fin de localizar su influencia en la salud mental de las mujeres privadas de libertad. El género, como argumentó Da$v^{(2,3)}$, es el dispositivo por medio del cual la propia noción de crimen se produce como masculina (negra, pobre y joven), normalizando la idea de que las instituciones masculinas son la regla $a^{(4)}$. Como afirma Añaños $^{(5)}$, la estructura de la cárcel está pensada más para los hombres, situación definida por el desconocimiento e indiferencia de los rasgos específicos de las mujeres ${ }^{(5,6,7)}$.

La centralidad del género en la producción y comprensión del castigo estatal tiene sus raíces históricas en el surgimiento y conso- lidación de la prisión como castigo moderno ${ }^{(2)}$. De este modo, mientras los hombres con comportamientos desviados se han definido como criminales, las mujeres desviadas han sido representadas como insanas ${ }^{(7,8,9)}$, o según Aniyar ${ }^{(10)}$ y Caponi ${ }^{(11)}$ : infractoras, pecadoras sexuales, brujas, ebrias, de vida desordenada o desobedientes. De ahí que, las prisiones han sido las instituciones de encierro custodial dominantes para el denominado control social duro $^{(12)}$ de la criminalidad masculina, mientras que los manicomios y hospitales psiquiátricos han cumplido el mismo papel para reformar la insanidad femenina, generalmente sexualizada (asociada al comportamiento sexual) y racializada (concebida de forma diferenciada para mujeres blancas y negras) ${ }^{(13,14)}$. La continuidad de la representación generizada como femenina de la insanidad/criminalidad permanece hasta hoy en las prisiones de mujeres, donde las drogas psiquiátricas siguen siendo distribuidas de manera más extensiva a esta población en comparación con los hombres en la misma situación ${ }^{(2)}$.

El panorama actual de las cárceles femeninas en Latinoamérica refleja también la vigencia de los efectos del carácter generizado del castigo estatal. Así, aunque la población femenina encarcelada continúa siendo menor que la masculina $(6,4$ mujeres presas por cada 100 hombres), el aumento en la criminalización femenina $(51,6 \%)$ es mucho mayor que el aumento de hombres presos $(20 \%)$. Las tasas desbordadas de crecimiento del encarcelamiento de mujeres responden a las políticas de drogas actuales que han dado lugar a su excesiva criminalización ${ }^{(15)}$.

En el caso específico de Brasil, la población presa de mujeres ha crecido sustancialmente $^{(16)}$. En el año 2000, había 5.601 mujeres bajo medidas de privación de libertad y, en 2016, el número aumentó a 44.721, de las cuales, el $43 \%$ estaba en condición de presas provisionales (cumplían pena privativa sin haber sido juzgadas) ${ }^{(17)}$. Se reportó un aumento del $698 \%$ en 16 años, siendo más del $60 \%$ de la población privada de libertad por delitos de drogas. Países como Argentina, Costa Rica, México y Perú presentan la misma tendencia ${ }^{(15)}$. 
Con todo, a pesar del aumento exagerado de mujeres presas, el perfil sociodemográfico de la población femenina privada de la libertad en la región continúa siendo el mismo: mujeres jóvenes, no blancas, madres solteras $(80 \%)$, en condiciones de pobreza y vulnerabilidad socioeconómica, bajos niveles de escolaridad y ocupación ${ }^{(18,19)}$. La criminalización y el encarcelamiento de estas mujeres se configura como una experiencia más de exclusión, que se suma al continuo de exclusiones que ya recaían sobre ellas desde antes de ser apresadas ${ }^{(9,20,21)}$. De este modo, las mujeres que están encarceladas por tráfico de drogas a menudo pertenecen a los eslabones más débiles de las estructuras criminales y realizan actividades de bajo nivel, pero de muy alto riesgo, como el transporte y comercialización de drogas a pequeña escala ${ }^{(19)}$. Su detención no ayuda a desmantelar los mercados ilegales de drogas, ni a mejorar la seguridad pública, ya que no están vinculadas a grandes redes criminales ni ocupan puestos de alto nivel o gerenciales y son fácilmente reemplazables. Ellas no representan un peligro real para la sociedad ${ }^{(15)}$ y su aprisionamiento, perpetúa un círculo vicioso de pobreza, vinculación a mercados de drogas y encarcelamiento ${ }^{(1,15,22)}$.

Además, el carácter generizado del castigo estatal se expresa en: condenas más altas que la de los hombres por los mismos delitos; asistencias carcelarias que ignoran las particularidades de las mujeres reclusas; y la precariedad de los espacios físicos de los establecimientos penitenciarios femeninos, los cuales, generalmente, son parte de las instalaciones de cárceles masculinas, no están habilitados para los fines destinados $\mathrm{y}$, a menudo, cuentan con muy poca inversión financiera ${ }^{(16,23,24,25)}$. A este panorama institucional, y frente a las necesidades particulares de las mujeres, se deben agregar las condiciones de hacinamiento, las malas condiciones sanitarias y de salud, el tiempo de inactividad y la violación sistemática de sus derechos (salud, estudio, trabajo, asistencia legal), situaciones que se traducen en la escasez de políticas públicas específicas para las mujeres presas ${ }^{(1,7,20,23,24)}$.
Esta compleja y alarmante situación de las prisiones femeninas, inevitablemente produce o perpetúa trastornos en la salud física y mental de las mujeres que, en muchos casos ya existían antes de la prisión, y que se tipifican como: ansiedad, depresión, insomnio, pánico, pensamientos suicidas, dependencia de drogas y alcohol, entre otros ${ }^{(16,26)}$. Por lo tanto, en ocasiones, los trastornos físicos y psicológicos derivados de las condiciones de vida previas al encarcelamiento se suman a los trastornos derivados de las condiciones de vida en prisión. Varios estudios han demostrado que el encarcelamiento aumenta el consumo de drogas psiquiátricas debido a los desequilibrios psicológicos y somáticos que se agudizan con el encierro ${ }^{(13,21,27,28,29)}$.

La extensión de la ingesta de medicación psiquiátrica intramuros en prisiones femeninas es una práctica cotidiana y normalizada en estas instituciones, a tal punto que ha sido poco visibilizada como una problemática social de salud mental y salud pública y como campo de discusión académica. En este sentido, el objetivo de este artículo es reconstruir y analizar desde una perspectiva etnográfica, los itinerarios y los significados de los procesos de medicalización psiquiátrica de las mujeres encarceladas en tres instituciones penales de mujeres en Brasil.

El artículo toma como encuadre analítico la perspectiva de la medicalización como proceso socio-histórico ${ }^{(30)}$. Esta noción fue definida por Conrad como un proceso que ocurre "cuando un problema es definido en términos médicos, es descripto usando un lenguaje médico, es comprendido a través de la adopción de un marco médico, o es tratado con una intervención médica"(30).

A su vez, de los estudios de la medicalización, se retoman dos conceptos que se desprenden y especifican dentro del proceso general, y permiten captar aspectos puntuales del mismo. Por un lado, la farmacologización, como proceso sociotécnico complejo, heterogéneo y dinámico, que involucra diferentes actores sociales, y consiste en recurrir a respuestas farmacológicas para los problemas de la vida. En este proceso, las condiciones humanas, sus capacidades y potenciales se 
convierten en "oportunidades para realizar intervenciones farmacológicas terapéuticas o de mejoramiento, sea de parte de los médicos, de los pacientes o de ambos" ${ }^{\prime \prime 11}$. Por otro lado, la patologización, que consiste en reducir los problemas de la vida a estados de morbidez.

Con esta estructura conceptual se busca captar diferentes matices, lógicas y sentidos que se verían reducidos y englobados de modo inexacto bajo el concepto de medicalización, con el que, sin embargo, se encuentran densamente relacionados. Por tanto, de acuerdo a lo expuesto, entendemos los itinerarios de farmacologización psiquiátrica como la práctica sistemática e institucionalizada del suministro/consumo de medicamentos psiquiátricos; suministro por parte de la institución penitenciaria femenina (en la figura de varios actores) y consumo por parte de la población penitenciaria durante la ejecución de su condena.

\section{METODOLOGÍA}

De acuerdo con el objetivo de la investigación, se llevó a cabo un estudio cualitativo de carácter etnográfico, basado en una concepción y práctica de producción de conocimiento que busca comprender los fenómenos sociales desde la perspectiva de sus miembros ("actores", "agentes" o "sujetos sociales"). Estos son informantes privilegiados, pues solo ellos pueden dar cuenta de lo que piensan, sienten, dicen y hacen con respecto a los eventos que los involucran ${ }^{(32)}$. De este modo, la presente etnografía se realizó dentro de tres cárceles femeninas brasileñas: la Penitenciaría Femenina del Distrito Federal (PFDF), Brasilia (desde octubre de 2006 a febrero de 2007); la Penitenciaría Industrial Estevão Pinto (PIEP), conocida como "Casa das Barbies", ubicada en el centro de Belo Horizonte (desde septiembre de 2007 a marzo de 2008); y el Presidio José Abranches Gonçalves (PJAG), también conocido como "Furacão", ubicado en Ribeirão das Neves, área metropolitana de Belo Horizonte, en el estado de Minas Gerais (desde septiembre de 2007 a marzo de 2008).
Aunque el trabajo de campo fue realizado hace algunos años atrás, según Strathern ${ }^{(33)}$, la etnografía se hace en el trabajo del tiempo, que transcurre entre el campo y la escritura del campo y que se actualiza con cada nueva forma de interrogarlo, analizarlo y representarlo. Además, estamos seguras de la vigencia y extrema relevancia de la problemática tratada en el contexto regional, donde la intersección entre los estudios de género, medicalización (especialmente su vertiente de la farmacologización de la sociedad) y las agencias de control sociopenal, ofrece posibilidades de análisis fructíferos y capaces de visibilizar una problemática compleja por excelencia, como es la que ocupa el artículo.

Las prisiones, al ser contextos pautados por relaciones explícitas de poder, control y vigilancia sobre los actores carcelarios como sobre las y los investigadores, le otorgan al trabajo de campo etnográfico intramuros algunas particularidades (en términos de desafíos, obstáculos y constantes negociaciones) que vale la pena resaltar: desde el acceso a las instituciones, los tiempos que demoran los jueces para dar el permiso, los tiempos autorizados para realizar el trabajo de campo dentro y los días y horarios de la semana permitidos para realizar las visitas, hasta los tránsitos por los espacios físicos y las interacciones sociales con las personas.

Así, para hacer trabajo de campo en estas cárceles, primero, se socializó la investigación con jueces penales de cada circuito donde estuviera adscrita la respectiva institución penal y se solicitó su autorización para el ingreso y ejecución de la investigación. Tras este permiso se presentó el trabajo a las respectivas directoras de los presidios y después a las directoras de las diferentes dependencias (educación, salud, resocialización, trabajo, dirección jurídica), así como a las guardias de seguridad. Solamente, después de todo ese recorrido pudimos finalmente encontrarnos y entrevistar a las mujeres privadas de libertad.

En la Penitenciaría Femenina de Brasilia, pese a la autorización del Juez de ejecuciones criminales, la directora de la Penitenciaria no autorizó nuestro ingreso. Aunque poco 
tiempo después tuvo que retractarse, acatar la orden del juez y dejarnos entrar. Así, las visitas, los tránsitos y las actividades previstas quedaron supeditadas a su voluntad, quien a su modo y poco a poco impidió la continuidad de la investigación en esa institución. Por ese motivo, nos desplazamos a Belo Horizonte, Minas Gerais, donde se nos abrieron generosamente las puertas de las instituciones penales mencionadas.

Entramos asiduamente durante seis meses, un día a la semana, en ambas instituciones penales femeninas. Considerando los contextos, contamos con condiciones favorables de investigación tales como: el ingreso y uso de la grabadora dentro de las instituciones, el derecho a la privacidad en las entrevistas con las internas; la disponibilidad de espacios físicos y guardias que permitían nuestras visitas; $y$, por último, contamos con un relativo tránsito por algunos espacios de las cárceles. Además, tuvimos la oportunidad de visitar la estación de policía durante una tarde, donde muchas mujeres comienzan de manera ilegal su privación de libertad, lo que nos permitió rastrear estos procesos de medicalización desde su inicio.

Aplicamos algunas técnicas de recolección de información propias de la etnografía como: observación participante, conversaciones informales, entrevistas semiestructuradas y relatos de vida. Tuvimos la oportunidad de entrevistar a diversos actores de las respectivas instituciones penales (mujeres privadas de la libertad de los regímenes cerrados y semiabiertos, directoras de instituciones penales, directoras de resocialización, de salud, trabajadores sociales, psiquiatras, enfermeros, profesores y guardias). La mayor parte de las entrevistas ocurrieron dentro de las prisiones y algunas (generalmente con los técnicos) fuera de estas. En general, las entrevistas tuvieron una duración de aproximadamente dos horas y fueron grabadas cuando las personas así lo permitieron. Cabe resaltar que las conversaciones informales también fueron una fuente de información muy importante.

En cuanto a la observación, participamos en las diferentes actividades diarias del cotidiano intramural, como en eventos ceremoniales que tuvieron lugar durante el trabajo de campo. También, tuvimos acceso a varios tipos de información institucional como registros de administración de medicamentos y algunos datos estadísticos de la población carcelaria para contextualizar el fenómeno de farmacologización psiquiátrica.

El trabajo etnográfico y las diferentes herramientas de recolección de información se orientaron a rastrear los itinerarios de suministro/consumo de medicamentos psiquiátricos intramuros. Enfatizamos en los significados, prácticas y relatos desde el punto de vista de los diferentes actores y sus implicaciones individuales e institucionales.

En relación con los aspectos éticos de la investigación, considerando las características del contexto, la población y la temática estudiada, es necesario resaltar que se aplicaron los protocolos éticos de la investigación social. A pesar de la condición de vulnerabilidad de los actores y de la naturaleza de los contextos propuestos para la realización de trabajo de campo, consideramos que los objetivos del proyecto y las experiencias sobre las que se indagó a los y las participantes no representaron riesgo o consecuencias negativas para su seguridad, dignidad personal, o proceso jurídico o institucional en el que se encontraban involucradas al momento de la investigación. Vale aclarar que, en los casos en que los contenidos de la información recogida comprometían de alguna manera a algunos de los participantes, tanto la recolección, el almacenamiento como los usos de la información obtenida en campo estuvieron orientados bajo parámetros de seguridad, confidencialidad y anonimato. Los nombres reales fueron cambiados por nombres ficticios para proteger la identidad de los participantes en la investigación. Además, hubo un vector reflexivo con el fin de aplicar los instrumentos de recolección de la información en sintonía con la situación de la población con la que se trabajó en los distintos contextos.

La participación de los informantes en la investigación fue de carácter voluntario, se garantizó el anonimato de su identidad y se les informó verbalmente los objetivos y metodología de la investigación, así como el 
uso estrictamente académico de la información. Por tanto, el número de participantes dependió principalmente de la participación voluntaria en la investigación. Asimismo, especialmente en lo referente a personas privadas de la libertad, el equipo aclaró que en ningún caso se comprometería a asumir la representación o asesoría legal o judicial de los participantes, ni al desempeño de tareas distintas a los propósitos de la investigación.

Con relación a la naturaleza y características de la investigación etnográfica, el proceso de consentimiento informado no es conveniente para ser implementado en este caso, tal y como se ha contemplado tradicionalmente (formal y escrito), dadas las características de los contextos carcelarios. Las personas pueden llegar a sentirse incómodas y desconfiadas al tener que firmar documentos escritos, ya sea porque no pueden leer o entender el documento, como también por las consecuencias negativas que les pudo haber generado firmar documentos en el pasado. En experiencias anteriores en contextos carcelarios, este instrumento fue interpretado por los interlocutores como un dispositivo que, en lugar de garantizar el anonimato y el compromiso del investigador frente al manejo responsable de la información, resultó intimidante, comprometedor y generador de desconfianza e inseguridad. Por estas razones, no se hizo uso del consentimiento informado escrito y formal, pero si se informó con un lenguaje simple y claro, reiterando el compromiso de brindar la información que era requerida.

\section{RESULTADOS}

A continuación, se presentan los procesos de medicalización psiquiátrica en el contexto carcelario, marcados por tres itinerarios: criminalización, patologización y farmacologización. Estos itinerarios pueden ser leídos como una de las diversas tecnologías de poder que actúan en diversas escalas y niveles sobre los cuerpos femeninos, desdoblándose dentro y fuera de los muros de las prisiones.
Los itinerarios son entendidos como las trayectorias que las mujeres inician con su encarcelamiento masivo, continúan una vez están privadas de la libertad con la patologización y consecuente farmacologización. Estos itinerarios se configuran en la intersección entre los diversos actores carcelarios, las instituciones penales en cuestión, la psicofarmacología intramural, los discursos (psiquiátricos, criminológicos, de seguridad y de género) y los significados que la farmacologización tiene para los involucrados.

\section{Patologización y farmacologización de los cuerpos}

Para comenzar, se considera importante resaltar la diferencia entre una persona que presenta síntomas de la "neurosis del confinamiento" tales como: ansiedad, depresión, angustia, insomnio, agitación y otras psicosomatizaciones causadas por la propia condición del aprisionamiento, y una persona con "trastorno psiquiátrico", cuyas raíces preceden la prisión o que se trastornan psíquicamente en este ambiente ${ }^{(27,34)}$. Esta diferencia es relevante a la hora de ponderar los efectos de un "tratamiento medicamentoso" en las personas presas que no son pacientes psiquiátricas, pero que por las condiciones del aprisionamiento y, justamente por la medicalización sistemática, pueden tornarse en algo regular.

En el momento de hablar con las mujeres, ellas manifestaban cómo continuamente les suministraban medicinas "para el sueño y la ansiedad". Muchas veces, esta medicación era administrada sin ninguna evaluación médica. Al hablar con algunos funcionarios y trabajadores de las cárceles se mencionó que este suministro, en la mayoría de ocasiones, puede verse como una práctica común e indiferenciada. Al respecto, una de las directoras de salud de las penitenciarías afirmó que el elevado suministro y consumo de medicamentos psiquiátricos dentro de las prisiones femeninas se explica, precisamente, como consecuencia de la no diferenciación, por parte de los técnicos de las diversas unidades 
prisionales, entre el trastorno psiquiátrico y la manifestación de los síntomas inherentes al confinamiento.

La secretaria comenzó a preguntar a las unidades penales qué estaban Ilamando de trastorno psiquiátrico. Para ellos, en una unidad con 396 presos, 100 de ellos tenían trastornos mentales por: síntomas depresivos, no están durmiendo, está agitado, nervioso. Eso no es trastorno psiquiátrico, eso es un proceso natural de la propia reclusión. (Sandra, personal de salud penitenciaria)

Como argumentan Davar ${ }^{(14)}$ y Veysey ${ }^{(35)}$, muchas de las situaciones clasificadas como trastornos de la personalidad representan respuestas a problemas situacionales antes que a condiciones patológicas. Sin embargo, las reclusas son patologizadas y se les administra medicación psicotrópica. El caso que una interna comenta ejemplifica lo que ocurre con muchas mujeres que, una vez presas, son tratadas farmacológicamente como enfermas psiquiátricas:

Ellos [médicos y guardias] dicen que ella es agresiva, pero ella no lo es porque en la calle ya la conocía y nunca había tomado remedios. Ella es súper normal, no es agresiva, son los remedios los que hacen que ella se vuelva medio lerda, que la hacen ponerse nerviosa. Esos remedios son tipo una droga, porque además de ser muy fuertes, modifican a las personas. (Diana, mujer privada de la libertad)

Así, según los datos proporcionados por la PFDF, en 2004, la enfermedad de mayor ocurrencia entre la población presa fue la depresión $(52 \%)$, seguido de la dependencia química $(49 \%)$. Isabela, una interna, nos relata las consecuencias que sufrió su salud mental con el cautiverio:

Yo estuve enferma aquí dentro, estuve en cama muy mal. Mi mamá hasta pensó que me iba a morir. Tuve depresión y síndrome de pánico. No podía salir de la celda, no podía hacer nada, se me congelaba todo el cuerpo, tiritaba y me ponía muy nerviosa, tenía mucho miedo, hasta los pasos de las personas me asustaban, cualquier cosa, ¿sabe? El ruido, la resonancia de alguien, la respiración fuerte de las personas, hasta los ronquidos me daban pánico. Yo me quedaba dormida a las cuatro de la mañana, cambiaba la noche por el día, me volví loca, de salir corriendo. Yo me jalaba el pelo de mi cabeza, me volví loca aquí dentro. Cambié el remedio varias veces pues tomo psicotrópicos, soy dependiente de ellos. Es un vicio, porque son seis años que ya Ilevo tomándolos, entonces ahora tomo de los más fuertes. (Isabela, mujer privada de la libertad)

Este relato muestra los efectos, sobre las mujeres, de la patologización y administración excesiva de psicofármacos a los cuerpos encarcelados. Refleja las formas cómo la prisión establece la patología y la dependencia a los medicamentos psiquiátricos creando, incluso, las condiciones para trastornos psiquiátricos reales frente a los procesos generados por el confinamiento. Así, queda en claro de qué modo el sistema penitenciario, de manera paradójica pero explícita, tipifica como enfermas a las personas que después trata a través de psicotrópicos, generando o perpetuando un círculo vicioso de farmacologización-patologización-farmacologización muy delicado. En síntesis, siguiendo a Kleinman ${ }^{(36)}$ y Gaines ${ }^{(37)}$, la cárcel es un espacio social configurado a partir de relaciones de poder, interacciones y relaciones sociales muy particulares, donde la enfermedad y la salud, además de fenómenos fisiológicos, son también respuestas individuales y sociales asociadas a las condiciones de aprisionamiento, por tanto, procesos socialmente construidos y producidos.

Así, la clasificación de las personas en sanas o enfermas no es una actividad pragmática basada exclusivamente en un hacer o saber médico. Esta categorización depende de los diversos actores, de las experiencias y 
de las dinámicas propias de los contextos. En este sentido, la construcción de los parámetros de salud/enfermedad de las mujeres reclusas emerge de los contextos en los cuales están inmersas.

\section{Actores e instituciones penales}

En el itinerario de la práctica de medicamentos psiquiátricos son múltiples los actores directamente involucrados en el suministro. A lo largo del trabajo de campo, se identificó que los equipos de salud mental, es decir, las y los profesionales de psiquiatría, medicina clínica, psicología y enfermería, dentro de las unidades carcelarias o fuera de estas, son quienes se vinculan a la atención de las internas y participan del itinerario. La composición de estos equipos varía según el tipo de institución carcelaria en que las internas estén cumpliendo su pena, esto es: estación de policía, presidio o penitenciaria.

Debido al carácter transitorio de las internas por las estaciones de policía, estas instituciones no tienen un equipo de salud que brinde atención a esta población dentro de sus instalaciones físicas. Estas unidades cuentan con una enfermera y cuando se requiere atención de urgencia se acude al hospital público más próximo. En los presidios, el equipo de salud mental que se encuentra dentro de las instituciones está formado por profesionales de medicina clínica, psicología y enfermería. Las atenciones psiquiátricas se realizan en el hospital más cercano. Las penitenciarías generalmente cuentan con la adecuación del espacio físico y la totalidad de los equipos de salud mental al interior de las instituciones. Solo en los casos de urgencia, las mujeres son llevadas y atendidas en los hospitales públicos más próximos.

En teoría, el único profesional habilitado para recetar los medicamentos psiquiátricos son los psiquiatras clínicos vinculados a las instituciones, los encargados de la salud mental de las mujeres. No obstante, en la práctica, dentro y fuera de las rejas, los médicos de diferentes formaciones o especialidades también recetan estos medicamentos.
En el caso de las estaciones de policía, no hay nadie que formule y las enfermeras simplemente los distribuyen. En el caso de los presidios donde las salidas para las atenciones están atravesadas por las dificultades del retiro transitorio, muchas veces es el médico clínico quien formula y retira los remedios. De la misma forma, en las penitenciarías, cuando el psiquiatra no está, es el médico clínico el encargado de la prescripción.

Con todo, en el cotidiano intramuros, las personas que tienen la responsabilidad directa del suministro de estos "remedios controlados" y de todos los medicamentos para las internas es el personal de enfermería. Son quienes los separan y preparan las dosis, que distribuyen y controlan, de manera más directa que las y los profesionales de psiquiatría y medicina, el suministro y la ingesta de estos medicamentos. Así, por ejemplo, en una de las cárceles estudiadas, una enfermera afirmó que, cuando una interna manifestaba el deseo de parar de consumir los medicamentos, tenía que firmar un acuerdo que comprobara su voluntad para poder parar de suministrarlos.

Las guardias también cumplen un papel destacado en esta práctica, pues son ellas, quienes, durante la noche, distribuyen los medicamentos que las enfermeras dejan preparados para las internas. También son quienes tienen el mayor contacto con las reclusas y las que están encargadas de la disciplina y la seguridad.

La presa pide, ella quiere medicamentos y si usted no atiende esa demanda, se genera un problema disciplinar, comienza a gritar, le pega a las paredes, pelea con las otras. En fin, no hay nada que hacer, está causando un montón de problemas en la celda, junto con otras doce presas que están en colchones en el piso. En esa situación los remedios ayudan. A veces, la ansiedad, la agresividad es tanta que las guardias le pedimos al médico que los prescriba. (Patricia, guardia penitenciaria)

En los procesos de la medicalización psiquiátrica de las mujeres, particularmente en los 
itinerarios de patologización y farmacologización, se muestra que el suministro de medicamentos psiquiátricos no es exclusivo de los profesionales de la salud e involucra otros actores (como las guardias), y otras justificaciones (seguridad y disciplina) que apuntan a la contención y control de la población femenina presa y no solamente a criterios médicos, como lo veremos a seguir.

\section{Significados de la farmacologización psiquiátrica}

En este itinerario de farmacologización psiquiátrica intramuros se constató que, por un lado, el suministro de medicamentos psiquiátricos es un mecanismo de contención institucional sobre la población presa para mantenerla más dócil y controlada, manteniendo la disciplina y seguridad. Por otro lado, la elevada demanda y consumo de estos medicamentos por parte de las internas actúa, simultáneamente, como un mecanismo de adaptación-resistencia que mitigan las tensiones y los dolores del aprisionamiento, que "matan" el penoso tiempo ocioso y que ayudan a escapar mental y temporalmente de la hostil realidad del encierro, tal como indica Ordóñez-Vargas ${ }^{(38)}$.

Como mecanismo de control institucional fue unánime la concepción de que en la prisión la práctica psiquiátrica es farmacéutica. Al respecto los psiquiatras afirmaron:

La psiquiatría en la institución es predominantemente psicofarmacológica, es decir que, nuestra función es hacer el relato del examen psicopatológico de las pacientes para dar un diagnóstico de su personalidad y los riesgos que ellas presentan para la sociedad. Es un trabajo hecho sobre la base de la cuestión de la criminología y la farmacología en cada institución. (Dr. Nelson, psiquiatra penitenciario)

La cárcel es el final de una secuencia de exclusiones familiares y sociales. En ese sentido, yo digo que la psiquiatría no medica las causas, medica las consecuencias. Mi función aquí dentro es básicamente realizar una contención medicamentosa. (Dr. Pedro, psiquiatra penitenciario)

La medicación es una camisa de fuerza química que es necesaria. Es una posibilidad de modificación del ser humano que está aquí para resocializarse. (Dr. Carlos, psiquiatra penitenciario)

La psiquiatría intramuros es psicofarmacológica y la respuesta farmacologizadora está más orientada a la contención y a la eliminación del síntoma que a tratar la raíz de los problemas. Sorprendentemente, para los psiquiatras, la medicación psiquiátrica debería ser todavía mayor, reflejando la prevalencia de criterios criminológicos positivistas que articulan discursos médicos y jurídicos ${ }^{(39)}$. Es así como se visualizan las formas en las que el género, como una categoría de poder, atraviesa la vida de las mujeres en la cárcel y las clasifica como criminales desviadas o enfermas, patologizándolas y medicándolas con el fin de controlarlas y transformarlas.

Para las empleadas de la administración, como para las guardias carcelarias, la medicación también ayuda a contener la población de forma efectiva, debido al número de guardias con relación a la población interna que custodian, como lo afirmó una de ellas:

...infelizmente con las condiciones tan
precarias que tenemos aquí dentro, a
veces, es preferible que las internas se
queden sin trabajar que tener alguna
fuga por la falta de guardias. (Carmen,
personal servicio penitenciario)

Algunos de estos profesionales afirman que:

...es mejor medicarlas y dejarlas en las celdas, que custodiarlas en los talleres profesionalizantes o en las laborterapias. (Luz, personal de servicio penitenciario)

En este sentido, el tiempo ocioso de las mujeres presas, que representa gran parte del día 
y toda la noche; tiempo en que ellas están encerradas en una celda, durante años, junto con otras doce o catorce mujeres, es uno de los factores que contribuye al alto y recurrente consumo de medicamentos. Se crea, de este modo, otro círculo vicioso referido al ocio-medicamento-ocio. Así, el ocio produce la necesidad del uso de los medicamentos y el exceso de estos impide a algunas internas poder hacer alguna actividad, además de quedarse todo el día en las celdas. El consumo excesivo de estos medicamentos sin actividades ocupacionales y físicas, sumado a la falta de acompañamiento psicológico y a las pésimas condiciones de aprisionamiento, se justifica principalmente por su función de contención y control, como afirmó una interna:

Hay chicas aquí dentro que no tienen la necesidad y sin embargo el psiquiatra les manda tomar. Hay muchas que los toman y se ponen peor, literalmente babean, parecen robots. Ellos lo que quieren es doparnos, que vegetemos. ¡Me parece una putada, es exagerado! (Lucía, mujer privada de la libertad)

Sin embargo, y a pesar de las tecnologías de control institucional, las internas crean mecanismos de adaptación-resistencia, retomando el concepto que Ordoñez-Vargas ${ }^{(38)}$ propone, que presuponen que, a pesar de las tecnologías de poder y control, en las márgenes existe una significativa capacidad de decisión y agencia por parte de las internas que se manifiesta, entre otras, en el consumo de medicamentos. Así, como mecanismo de adaptación-resistencia, para una amplia mayoría de internas que afirmaron la imposibilidad de "cumplir cana [condena] con la cara limpia", los medicamentos son un mecanismo de escape, un bálsamo a la dura realidad del encierro.

Estos mecanismos se manifiestan en múltiples estrategias que las mujeres usan para conocer, obtener y consumir los medicamentos. Entre estas estrategias encontramos: formas particulares de relacionarse y comunicarse con los psiquiatras para obtener las prescripciones (síntomas que deben decir que tienen); formas de control a partir de los comportamientos, como gritos, Ilantos y diferentes expresiones de agitación y exaltación, para que enfermeros y guardias les suministren medicación; y formas de obtención de los medicamentos relacionadas con la compra y venta de comprimidos entre las mismas presas o como moneda de cambio.

Subordinadas y limitadas a las tecnologías de poder y control y, al mismo tiempo, en coexistencia negociada, estos mecanismos implican una constante, delicada y tensa negociación entre poder, sumisión, resistencia, agencia y sobrevivencia. Así, los múltiples matices que la práctica de la administración de remedios psiquiátricos adquieren, delinean las formas cómo las mujeres presas usan y crean ambivalentes, híbridos e indiscernibles mecanismos de "adaptación-resistencia", para sortear los extremos estados de represión y confinamiento a los que se encuentran sometidas: para anestesiar el alma y la cabeza; para dormir durante largos períodos de tiempo y apaciguar la tristeza, la ansiedad, las insatisfacciones y mortificaciones profundas del encierro.

\section{Esbozo sobre la psicofarmacología intramuros}

Con la finalidad de contextualizar la psicofarmacología carcelaria, realizaremos un esbozo sintético sobre los medicamentos psiquiátricos más consumidos y suministrados en las diversas instituciones carcelarias, según las internas; su clasificación por grupo de medicamento; sus efectos "terapéuticos" y colaterales; $y$, los usos que los diversos actores les atribuyen.

En las estaciones de policía en las que, aunque de forma ilegal, las mujeres son retenidas e inician el cumplimiento de la privación de la libertad por falta de cupos en las cárceles, se suministra el "nitrio" en las comidas. El nitro es el nombre usado por las mujeres en diversas instituciones penales femeninas para designar lo que ellas identifican como la primera sustancia controladora que les proporciona el sistema a través de la comida, dejándola rojiza. Según Lucía, una interna: 
...el "nitrio" lo usan para quitarnos las ganas de tener sexo, el "nitrio" nos deja todas hinchadas, gordas como vacas. Ese es el primer veneno que nos da el sistema. (Lucía, mujer privada de la libertad)

Debido a que en las estaciones de policía entran más fácilmente drogas ilícitas en comparación con las cárceles, el uso de medicamentos psiquiátricos tiende a ser menor. Ya las instituciones penales ofrecen una variedad mayor de medicamentos que incluyen desde analgésicos como la dipirona sódica para los múltiples dolores, pasando por medicamentos para la hipertensión, la diabetes, la tuberculosis, la hepatitis, el VIH y, los antedichos medicamentos psiquiátricos.

Los principales medicamentos psiquiátricos se dividen en cuatro grupos: ansiolíticos, antidepresivos, antipsicóticos y estabilizadores del ánimo como los antiepilépticos. Los ansiolíticos benzodiazepínicos, también conocidos como tranquilizantes, sedantes o calmantes, al actuar en el sistema nervioso central, ejercen una acción selectiva sobre la ansiedad $^{(40)}$.

En las instituciones carcelarias femeninas estudiadas en Belo Horizonte y en Brasilia se suministraban los siguientes ansiolíticos durante la investigación: diazepam (10 mg) y clonazepam (Rivotril ${ }^{\circ}$ ) (2 mg). Los efectos y reacciones de los medicamentos son diversos en cada mujer. Por ejemplo, María "ama" el diazepam porque le da una "onda agradable", describió cómo la ayudaba a relajarse y a calmarse. Para Amanda, el Rivotril ${ }^{\circ}$ es como la cocaína, la deja acelerada, despierta, con la adrenalina a flor de piel.

Los antidepresivos, que corresponden al segundo grupo mencionado, son medicamentos cuyos efectos terapéuticos consisten en aumentar el tono psíquico, mejorando el humor $y$, consecuentemente, mejorando el desempeño psíquico de manera general. La acción terapéutica de los antidepresivos tiene lugar en el sistema límbico, el principal centro de las emociones ${ }^{(40)}$. En las dos prisiones de Belo Horizonte se suministra amitriptilina (25 mg) e imipramina (25 mg), que pertenecen al grupo de los antidepresivos tricíclicos, correspondientes a las primeras generaciones de estos medicamentos, cuyas fórmulas químicas ya estaban obsoletas y contraindicadas. En Brasilia, se suministraba amitriptilina (25 mg); también fluoxetina (20 mg), el cual ya es un antidepresivo más reciente.

En el tercer grupo se encuentran los antipsicóticos o neurolépticos. Antipsicótico es un término genérico aplicado de manera amplia a diversas clases de químicas de drogas, empleadas en el manejo sintomático de varias condiciones psicóticas, especialmente, la esquizofrenia y los estados de euforia. Son medicamentos inhibidores de funciones psicomotoras, que atenúan los disturbios neuropsíquicos como los delirios y las alucinaciones $^{(40)}$. En Belo Horizonte se suministraban los siguientes medicamentos pertenecientes a este grupo: haloperidol (Haldol ${ }^{\circ}$ $5 \mathrm{mg}$ ), levomepromazina (Neozine 100 y 25 mg) y carbonato de litium. En Brasilia, además de los anteriores, también se suministraba risperidona ( $2 \mathrm{mg}$ ). El consumo de la mayoría de estos medicamentos provoca reacciones que algunas reclusas describen como incómodas y fuertes. En palabras de una mujer:
Yo tomaba nervozin 100 [refiriéndose de modo jocoso a Neozine ] que es para dormir; amitriptilina que es anti- depresivo y un diazepam. El primero, pedí a los médicos que lo suspendieran porque me dejaba tirada en una cama, uno no tiene reacción ni para levantarse. También, les pedí que suspendieran la amitriptilina y quedarme solo con el diazepam porque veo lo que sucede a mi alrededor. ¡Con los otros no me doy cuenta de nada! (María, mujer privada de la libertad)

Para finalizar, en el cuarto grupo están los estabilizadores del ánimo: los antiepilépticos o anticonvulsivos (que evitan las crisis epilépticas) y el litio. Según el relato de Soraya, quien tomaba con regularidad un antiepiléptico, cuenta cómo un día se levantó para ir al baño y simplemente, se cayó, el cuerpo no le respondió. 
Aunque contamos con los datos oficiales de las diversas instituciones, estos no revelan las cantidades reales que las internas consumen, porque ese número no considera el comercio informal de estos medicamentos entre la población carcelaria, ni cuenta las drogas ilícitas que también son consumidas. De acuerdo a los reportes de las cárceles se encuentra que, en la Presídio Feminino Estevão Pinto, el consumo mensual de medicamentos durante el mes de septiembre de 2007 fue de 6.380 comprimidos para una población de 379 mujeres. De estos, 2.010 eran antidepresivos, 960 antipsicóticos, 890 ansiolíticos y 2.070 antiepilépticos. En el Presidio José Abranches Gonçalves durante el mes de abril de 2007, la población carcelaria formada por 132 internas consumió un total de 8.720 comprimidos, siendo 3.100 antidepresivos, 720 antipsicóticos, 1.050 antiepilépticos, 240 anticolinérgicos y 3.610 ansiolíticos.

A pesar de no contar con información estadística más precisa, el elevado consumo de medicamentos psiquiátricos por las mujeres se expresa también en el Programa Individualizado de Resocialización (PIR), suministrado por el Presidio José Abranches Gonçalves, en el que aparecen las prescripciones médicas de las internas. Llama la atención el suministro de dos medicamentos distintos del mismo grupo como, por ejemplo:

Marilou: usa Gardenal, Diazepam, Rivotril, Haldol, Triptanol y Akinedon. (1 antiepilético, 2 ansiolíticos, 1 antipsicótico, 1 anticolinérgico).

Graziele: usa Neozine, Haldol y Gardenal. (2 antipsicóticos y 1 antiepiléptico).

Soraia: usa Neozine, Haldol, Tegretol. (2 antipsicóticos y 1 anti-epiléptico).

Elisângela: usa diazepam, Neozine, Haldol, Gardenal. (1 ansiolítico, 2 antipsicóticos y 1 anti-epiléptico).

Claudia: usa 3 Triptanol por la mañana y 3 Triptanol por la noche.

Delma: usa Neozine, Imipramina, Tegretol, Akineton. (1 antipsicótico, 1 antidepresivo, 1 anti-epilético y 1 anticolinérgico).

Jeruza: usa Haldol y Neozine. (2 antipsicóticos).
Eliete: rechaza tomar Haldol y cualquier otro medicamento.

La administración de drogas psiquiátricas en las prisiones femeninas tiene un carácter muy variado y oscilatorio, sujeto a diversos factores. Así, por ejemplo, las internas que recién llegan a las estaciones de policía o a las instituciones penales y están en la sección de celdas aisladas (entre 15 a 30 días) toman medicamentos durante ese periodo. Las internas que van a las celdas de castigo también consumen una elevada cantidad de remedios durante el tiempo que permanecen allá. Los diversos eventos (malas noticias con los hijos y familiares allá afuera o sobre su situación jurídica) también generan mucha impotencia y ansiedad, haciendo que tanto las internas como las instituciones acudan a los medicamentos. Del mismo modo, las internas que son separadas de sus hijos y de la guardería y pasan nuevamente a la convivencia con las otras internas, son altamente medicadas para poder soportar la dolorosa ruptura. En ese sentido, tanto la entrada a la cárcel como su salida son períodos denominados "curva $u$ ", pues es cuando se manifiestan los picos de mayor ansiedad y consumo de medicamentos $^{(38)}$. Frente a esto, una mujer afirma:

Consumo por la mañana, por la tarde y por la noche. Consumo Neozine, Amitriptanol, Rivotril y hay otro que no sé el nombre. Ahora no quiero que me los corten porque acabé de perder a mi bebé, una niña de cinco años. No estoy preparada para aceptar eso de "cara limpia", porque esos remedios me ayudan a no pensar, a viajar, a estar volando, lenta, leve, suelta. Sin consumir nada, no sé cómo voy a reaccionar frente a esta situación. (Paula, mujer privada de la libertad)

Estos fragmentos muestran que, independientemente del carácter variado, oscilatorio e intermitente del consumo a lo largo de su estadía en prisión, la farmacologización psiquiátrica es una práctica que forma parte de la vida cotidiana de las mujeres privadas de 
su libertad. Para las reclusas, ayuda a escapar de la hostil realidad y para los diversos actores institucionales sirve como una camisa de fuerza química, una técnica muy refinada y aséptica de represión, control y "reforma" de los cuerpos femeninos encarcelados a través de los medicamentos.

\section{DISCUSIÓN Y CONCLUSIONES}

Este artículo buscó reconstruir y analizar, desde una aproximación etnográfica, los procesos de medicalización psiquiátrica rastreados a partir de los itinerarios de la práctica sistemática de criminalización, patologización y farmacologización de mujeres en tres cárceles femeninas de Brasil. Para lograr nuestro objetivo, establecimos un diálogo interdisciplinar entre los estudios de género ${ }^{(2,3,9,18,41,42)}$, la antropología de la salud mental ${ }^{(14,36,37)}$ y la antropología del confinamiento ${ }^{(1,43,44,45,46)}$. A partir de los hallazgos en campo y la elección de una metodología que nos permitió vivir de manera directa e íntima la realidad de las mujeres privadas de la libertad, pudimos entender un fenómeno que, no solo habla de la realidad intramural (de Brasil y Latinoamérica, en general), sino también se refiere a la situación de muchas mujeres en la sociedad libre.

Siguiendo a Prado De Carvalho y Mayorga $^{(18)}$, Carvalho y Dimenstein ${ }^{(47)}$ y Almeda y Di Nella ${ }^{(7)}$, en nuestro caso fue evidente la posición de destaque de las mujeres con respecto al uso indiscriminado de estos medicamentos psiquiátricos, situación que en todo el mundo se convierte en un complejo problema de salud pública que alcanza grandes dimensiones. A partir de los relatos de las mujeres reclusas se hizo explícito que estos itinerarios comienzan desde antes de estar en la prisión y, están vinculados a la criminalización masiva y al encarcelamiento de mujeres en situaciones de desigualdad estructural (económica, cultural, social, política, jurídica). Continúan cuando, una vez encarceladas y bajo la etiqueta de criminales, las mujeres son patologizadas e insertadas en una práctica sistemática e institucionalizada de medicación psiquiátrica, que no termina con el final del encarcelamiento y ciertamente tampoco contribuye a la resocialización o "reforma" de las mujeres para la vida en sociedad.

La influencia del género en la criminalización femenina se expresa en los discursos criminológicos que todavía entienden y definen a las "delincuentes" como desviadas, inmorales y enfermas que necesitan ser reformadas o modificadas para encajar en lo esperado de una mujer. Esta connotación permite justificar la patologización y farmacologización de las mujeres. Este hallazgo se une al reportado en estudios recientes como los de Santos et al. ${ }^{(17)}$, Figueiró et al. ${ }^{(27)}$ y Dewa et al. ${ }^{(48)}$, quienes coinciden en considerar la medicación como un problema de las prisiones, relacionado con ser una "solución rápida" para mantener bajo control el ambiente en las cárceles, y ser un mecanismo para la psiquiatrización de las reacciones psíquicas a eventos vitales como las que atraviesan las mujeres en el entorno penitenciario.

Con respecto a la influencia del género en el diagnóstico de enfermedades mentales en las mujeres, siguiendo a Lagarde ${ }^{(9)}$, Caponi $^{(11)}$ y $\operatorname{Ruiz}^{(49)}$, la patologización femenina se ve impulsada por la concepción estereotipada de las mujeres que generalmente están catalogadas como histéricas, locas, emocionales o mentales, lo que también justifica de esta manera la patologización y la farmacologización psiquiátrica de las mujeres privadas de libertad. Del mismo modo, en un contexto abierto, cuando las mujeres visitan a un psicólogo o psiquiatra, entienden el malestar en términos de disfunción individual cuya respuesta es corregirla y eliminar los síntomas $^{(14,35,42)}$. Frente a esta patologización femenina, algunas feministas acusan a la psiquiatría y la psicología (disciplinas centrales de la salud mental), como responsables de la psicopatología de las mujeres al atribuir las causas de sus problemas a una esencia femenina, en lugar de comprender sus síntomas, causas y contextos ${ }^{(3,14,20,39,40,43)}$. Desde estas posturas feministas críticas se asume que las enfermedades mentales son producto del contexto social, económico y político que oprime a las mujeres, legitimando la 
patologización femenina y en este caso haciendo más aguda y violenta la experiencia de encarcelamiento.

Al proponer la categoría de itinerario de farmacologización psiquiátrica, apuntamos a un concepto que puede explicar, desde las vivencias de las mujeres, que el elevado consumo de medicamentos psiquiátricos en las prisiones está vinculado, no solo a trastornos psiquiátricos coincidentes, sino también a la patologización y a la sobremedicación institucional de las respuestas emocionales y psicosomáticas de las mujeres por parte de los dispositivos médicos y criminológicos que, en muchas ocasiones, terminan por producir la enfermedad que posteriormente se medicaliza. La farmacologización de las mujeres privadas de libertad se presenta como una gran paradoja desde la perspectiva de las políticas de salud mental, penal y de seguridad pública: un psicotrópico ilícito se reemplaza indistintamente por otro lícito (medicamento) y viceversa. Esta situación paradójica perpetúa o crea adicción a las drogas y, por lo tanto, como es explicado también por la Washington Office on Latin America ${ }^{(15)}$, mantiene esta cadena que alimenta ciclos de pobreza, mercado y consumo de drogas y encarcelamiento masivo.

Los significados de los itinerarios de farmacologización psiquiátrica para los diversos actores responden, para la institución, a un dispositivo de control relativo a la contención y "seguridad" de las instituciones penales sobre la población presa. Para las mujeres privadas de la libertad, la farmacologización psiquiátrica actúa como un eficaz dispositivo de adaptación-resistencia y sobrevivencia a los dolores del confinamiento ${ }^{(38)}$. Y, para el personal de salud, significa una respuesta más orientada a la contención de síntomas que la raíz de los problemas. Así, la farmacologización psiquiátrica, entre otras y numerosas prácticas de opresión en las prisiones femeninas emanan de sistemas penitenciarios históricamente configurados a través de categorías de masculinidad ${ }^{(2,50,51,52)}$, que revelan el carácter profundamente generizado del castigo estatal que, a su vez, refleja y profundiza la estructura generizada de toda la sociedad ${ }^{(3)}$

A partir de los elementos expuestos, en este trabajo se enfatiza en la necesidad de contextualizar y tratar la salud mental como un problema de sufrimiento social y no solo individual, que tiene una base sólida en la desventaja estructural oculta en las categorías médicas que intentan individualizar y medicar este problema de salud pública. Las políticas dirigidas a la salud mental de las mujeres (en prisión o en otros espacios sociales) deben comprender que los efectos que sufren las mujeres derivan de problemas sociales y estructurales, que vinculan las desigualdades de género, clase y raza, entre otras categorías de diferenciación.

En este sentido y para finalizar, nos unimos a la visión crítica ${ }^{(2,4,8,9)}$ que busca politizar y articular la salud mental de las mujeres con una comprensión del contexto social patriarcal, la distribución desigual del poder, los patrones socioculturales en las relaciones entre hombres y mujeres, y las formas en que estos patrones producen enfermedades. Solo desde estas integraciones será posible abogar por la salud mental y contribuir a la discusión sobre la influencia del género en la producción de enfermedades mentales y su consideración como un problema de salud pública cuyas dimensiones han sido subestimadas $^{(7,13,26,35)}$.

\section{AGRADECIMIENTOS}

Queremos agradecer al Conselho Nacional de Ciência e Tecnologia do Brasil por su apoyo financiero para el desarrollo del proyecto denominado: "As mulheres e a pena privativa da liberdade", vinculado a la convocatoria "Mulheres, gênero e feminismos", Edital 045/2005. La investigación fue financiada y realizada desde octubre de 2006 hasta octubre de 2008 y es con base en esta desde donde surgen los resultados y análisis presentados en este documento.

\section{REFERENCIAS BIBLIOGRÁFICAS}

1. Ordoñez-Vargas L. Gênero e etnografia: reflexões desde algumas prisões brasileiras. Cadernos Pagu. 2019;(55):1-31. doi: 10.1590/18094449201900550008. 
2. Davis A. Cómo el género estructura el sistema carcelario. En: ¿Son obsoletas las prisiones? Córdoba: Bocavulvaria Ediciones; 2017. p. 71-98.

3. Davis A, Dent G. A prisão como fronteira: uma conversa sobre gênero, globalização e punição. Revista Estudos Feministas. 2003;11(2):523-531. doi: 10.1590/ S0104-026X2003000200011.

4. Zaffaroni ER. El discurso feminista y el poder punitivo. En: Las trampas del poder punitivo. Buenos Aires: Biblos; 2000. p. 19-30.

5. Añaños Bedriñana FT, Yagüe Olmos C. Educación social en prisiones: planteamientos iniciales y políticas encaminadas hacia la reinserción desde la perspectiva de género. Pedagogía Social Revista Interuniversitaria. 2013;(22):1-6.

6. Yagüe Olmos CY. Mujeres en prisión. Intervención basada en sus características, necesidades y demandas. Revista Española de Investigación Criminológica REIC. 2007;5(4):1-24.

7. Almeda Samaranch E, Di Nella D. Mujeres y cárceles en América Latina: Perspectivas críticas y feministas. Papers: Revista de Sociología. 2017;102 (2):183-214.

8. Salinas C. Las cárceles de mujeres en México: espacios de opresión patriarcal. Iberofórum. 2014;IX(117):1-27.

9. Lagarde M. Los cautiverios de las mujeres, madresposas, monjas, putas, presas y locas. México DF: UNAM; 2006.

10. Aniyar de Castro L. Las mujeres infractoras: Impacto y amplificación de los efectos de la pena. Capítulo Criminológico. 2002;30(4):333-351.

11. Caponi S. Scientia Sexualis: el lugar de la mujer en la historia de la psiquiatría. En: Miranda M, (comp.) Las Locas: Miradas interdisciplinarias sobre género y salud mental. La Plata: Edulp; 2019. p. 19-48.

12. Pitch T. ¿Qué es el control social? Delito y Sociedad. 1996;1(8):51-72. doi: 10.14409/dys.v1i8.5757.

13. Banks C. Women in prison: a reference handbook. Santa Barbara: ABC-CLIO; 2003.

14. Davar BV. Women, society and mental illness. En: Mental health from a gender perspective. Thousand Oaks: Sage Publications; 2001.

15. Washington Office on Latin America, International Drug Policy Consortium, Dejusticia, CIM, Organización de los Estados Americanos. A guide for policy reform in Latin America and the Caribbean [Internet]. 2016 [citado 22 ago 2019]. Disponible en: https://tinyurl.com/ yxce8sjv.

16. Moura TW, Ribeiro NT. Levantamento Nacional de Informações Penitenciárias (Infopen). Brasília: Departamento Penitenciário Nacional, Ministério da Justiça; 2014.

17. Santos MV, Alves VH, Pereira AV, Rodrigues DP, Marchiori GRS, Guerra JVV. Saúde mental de mulheres encarceradas em um presídio do Estado do Rio de Janeiro. Texto Contexto - Enfermagem. 2017;26(2):e5980015. doi: 10.1590/0104-07072017005980015.

18. Prado De Carvalho D, Mayorga C. Contribuições feministas para os estudos acerca do aprisionamento de mulheres. Revista Estudos Feministas. 2017;25(1):95112. doi: 10.1590/1806-9584.2017v25n1p99.

19. Lima-Silva S. Mulheres e criminalidade: aspectos de uma inclusão enviesada. Revista Transgressões. 2017;5(2):104-118.

20. Almeda E. Mujeres encarceladas. Barcelona: Ariel; 2003.

21. Carlen P, Worrall A. Analysing women's imprisonment. Cullompton: Routledge, Willan Publishing; 2004.

22. Pereira M. Women and drugs: Destruction by incarceration. Hecate. 2002;28(1):154-162.

23. Almeda E. Pasado y presente de las cárceles femeninas en España. Sociológica Revista de Pensamiento Social. 2005;(6):75-106.

24. Mapelli B, Herrera M, Sordi B. La exclusión de las excluidas: ¿Atiende el sistema penitenciario a las necesidades de género?: una visión andaluza. Estudios Penales y Criminológicos. 2013;(33):59-95.

25. Antony C. Mujeres invisibles: las cárceles femeninas en América Latina. Nueva Sociedad. 2007;(208):73-85.

26. Pulido F, Rodríguez J, Colorado M. Factores sociodemográficos asociados con los síntomas depresivos en una muestra de mujeres recluidas en dos prisiones de México. Revista Panamericana de Salud Pública. 2009;26(3):209-215.

27. Figueiró RA, Dimenstein M, Alves D, Medeiros G. Consumo de medicação psicotrópica em uma prisão feminina. Revista Psicología Política. 2015;15(34):531-546.

28. Fazel S, Hayes AJ, Bartellas K, Clerici M, Trestman $R$. The mental health of prisoners: a review of prevalence, adverse outcomes and interventions. Lancet Psychiatry. 2016;3(9):871-881. doi: 10.1016/S22150366(16)30142-0.

29. Solomon A, Mihretie G, Tesfaw G. The prevalence and correlates of common mental disorders among prisoners in Addis Ababa: an institution based cross-sectional study. BMC Research Notes. 2019;12(1):394. doi: 10.1186/s13104-019-4425-7.

30. Bianchi E. ¿De qué hablamos cuando hablamos de medicalización?: Sobre adjetivaciones, reduccionismos y falacias del concepto en ciencias sociales. Revista Latinoamericana de Metodología en Ciencias Sociales. 2019;9(1):e052. doi: 10.24215/18537863e052.

31. Bianchi E. Saberes, fármacos y diagnósticos: Un panorama sobre producciones recientes en torno a la farmacologización de la sociedad. Psicología, Conocimiento y Sociedad. 2018;8(2):147-175. doi: 10.26864/ pcs.v8.n2.11. 
32. Guber R. La etnografía: Método, campo y reflexividad. Buenos Aires: Siglo XXI Editores; 2001.

33. Strathern M. O efeito etnográfico e outros ensaios São Paulo: Cosac Naify; 2014.

34. Varela-González O, Algora-Donoso I, GutiérrezBlanco M, Larraz-Pascual ME, Barreales-Tolosa L, Santamaría-Morales A. Uso de psicofármacos en prisión (CP Madrid III). Revista Española de Sanidad Penitenciaria. 2012;9(2):38-46.

35. Veysey B. Mental health issues of incarcerated women. En: Levin BL, Becker MA, (eds.). A public health perspective of women's mental health. New York: Springer; 2010. p. 245-260.

36. Kleinman A. Rethinking psychiatry: from cultural category to personal experience. New York: Free Press; 1988.

37. Gaines AD, (ed.). Ethnopsychiatry: the cultural construction of professional and folk psychiatries. Albany: State University of New York Press; 1992.

38. Ordóñez-Vargas L. Mujeres encarceladas: proceso de encarcelamiento en la penitenciaría femenina de Brasilia. Universitas Humanística. 2006;(61):183-199.

39. Foucault M. Historia de la locura en la época clásica II. México DF: Fondo de Cultura Económica; 2015.

40. Semple D, Smyth R. Oxford handbook of psychiatry. 3rd ed. Oxford: Oxford University Press; 2013.

41. Almeda Samaranch E. Criminologías feministas, investigación y cárceles de mujeres en España. Papers: Revista de Sociología. 2017;102(2):151-181.

42. Vindhya U. De lo personal a lo colectivo: cuestiones psicológicas y feministas de la salud mental de las mujeres. Revista de Estudios de Género: La Ventana. 2002;2(16):7-35.
43. Cunha M. The ethnography of prisons and penal confinement. Annual Review of Anthropology. 2014;43:217233.

44. Cunha M, Granja R. Gender asymmetries, parenthood and confinement in two Portuguese prisons. Champ Pénal. 2014;11:1-14. doi: 10.4000/champpenal.8809.

45. Palomar V. Maternidad en prisión. México DF: Universidad de Guadalajara; 2007.

46. Comfort M. Time together: Love and family in the shadow of the prison. Chicago: Chicago University Press; 2008.

47. Carvalho LF, Dimenstein M. O modelo de atenção à saúde e o uso de ansiolíticos entre mulheres. Estudos de Psicologia (Natal). 2004;9(1):121-129.

48. Dewa LH, Hassan L, Shaw JJ, Senior J. Insomnia management in prisons in England and Wales: a mixed-methods study. Journal of Sleep Research. 2017;26(3):322-329. doi: $10.1111 /$ jsr. 12503

49. Ruiz CO. Las mujeres y la enfermedad mental. Una perspectiva de género a través de la historia contemporánea. Cuadernos KÓRE. 2012;1(4):208-223.

50. Carvalho DT, Mayorga C. Contribuições feministas para os estudos acerca do aprisionamento de mulheres. Revista Estudos Feministas. 2017;25(1):99-116. doi: 10.1590/1806-9584.2017v25n1p99.

51. Carvalho ML, Valente JG, Assis SG, Vasconcelos AGG. Perfil dos internos no sistema prisional do Rio de Janeiro: especificidades de gênero no processo de exclusão social. Ciência \& Saúde Coletiva. 2006;11(2):461471.

52. Teixeira MA, Silva NM. Mulheres encarceradas: a saúde atrás das grades. Revista Psicologia, Diversidade e Saúde. 2017;6(4):324-339. doi: 10.17267/2317-3394rpds. v6i4.1708.

FORMA DE CITAR

Ordoñez-Vargas L, Cortés-García CM. Medicalización psiquiátrica en tres prisiones femeninas brasileras: un abordaje etnográfico sobre los itinerarios de criminalización, patologización y farmacologización. Salud Colectiva. 2020;16:e2507. doi: 10.18294/sc.2020.2507.

Recibido: 30 ago 2019 | Versión final: 10 jun 2020 | Aprobado: 15 jul 2020 | Publicado en línea: 20 ago 2020

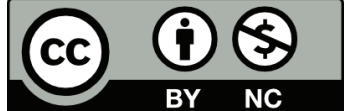

Esta obra está bajo una licencia de Creative Commons Reconocimiento-NoComercial 4.0 Internacional. Reconocimiento - Permite copiar, distribuir y comunicar públicamente la obra. A cambio, se debe reconocer y citar al autor original. No Comercial - Esta obra no puede ser utilizada con finalidades comerciales, a menos que se obtenga el permiso. 